a cursor to control a second well, which they use to collect the sloshing liquid and take it back to a base. The liquid behaves according to the laws of quantum mechanics rather than like an actual bucket of water - for example, to pick up the liquid, players can get it to 'quantum tunnel' from one well to another, something that players must learn to adapt to. Once they find ways to transfer the liquid, a computer can then convert their mouse movements to solutions to the real-world quantum egg box.

Sherson's team got around 300 people to play this level a total of 12,000 times on a volunteerresearch platform called ScienceAtHome. The researchers then fed the human solutions into a computer for further refinement. Not only were more than half of the human-inspired solutions more efficient than those produced by just computer algorithms, but the two best hybrid strategies were faster than what the quickest computers had been able to achieve working alone. "I was completely amazed when we saw the results," says Sherson.

\section{HUMAN ADVANTAGE}

What abilities humans bring to the mix is unclear. Although an interest in physics seems to correlate with ability in the game, success did not correlate with years spent studying quantum physics. Sherson suggests that the superior human strategies stem from the mind's ability

to capture the essence of a problem. Quantum concepts may seem less bizarre to people in a game than they do in other contexts, because it is an environment in which they expect rules to be broken, adds Sabrina Maniscalco of the Turku Centre for Quantum Physics in Finland, who runs an event aimed at making games that might benefit quantum physics.

\section{"We should try to be more spontaneous and intuitive about problem solving."}

To Sherson, the results also suggest that physicists could use their own intuition more. "We should try to be more spontaneous and intuitive about problem solving," he says. To that end, his team is building a version of the game in which physicists can tweak the scenario to represent different set-ups, potentially offering them new insights into their work.

Other quantum physicists agree that the finding that people can develop an intuition for quantum processes is surprising, but think that scientists already use intuition to solve quantum problems, at least at the mathematical level. By playing the game, people perhaps gain a form of that intuition, says Seth Lloyd, a physicist at the Massachusetts Institute of Technology in Cambridge. He notes that before babies learn to expect an object to stay where it is, they have a form of quantum intuition, which they lose.
"Before three months, if it disappears, they guess that's just how things are in the world. After three months, they think, 'Where'd the toy go'?"

Lloyd also says that much of the success of Quantum Moves is due to its clever design, which successfully translates a quantum problem to a visual one, but which could fail with more-complex quantum problems.

Physicists who are trying to develop quantum-computing algorithms already play around with graphical interfaces to help them to improve on existing solutions, says Charles Tahan, a theoretical physicist at the University of Maryland in College Park.

But Tahan does think that teaching quantum intuition through games has benefits. He has developed another game, Meqanic, that gets players to perform basic quantum computations and intuit the rules as they play. He hopes that it could boost student's abilities and help to find individuals who have an untapped natural flair for the field.

\section{CORRECTION}

In the News story 'Controversial darkmatter claim faces ultimate test' (Nature 532, 14-15; 2016), the last paragraph was amended to better reflect Katherine Freese's views on the DAMA collaboration's results. 